\title{
A NOTE ON THE DU BOIS-REYMOND EQUATIONS IN THE CALCULUS OF VARIATIONS
}

\author{
WILLIAM T. REID
}

1. Introduction. McShane [2] ${ }^{1}$ and Tonelli [4] have given conditions on the integrand function which insure that every absolutely continuous solution of the problem of minimizing the integral

$$
I[y]=\int_{x_{1}}^{x_{2}} f\left(x, y, y^{\prime}\right) d x
$$

satisfies the du Bois-Reymond form of the Euler equations. The principal purpose of the present note is to give an alternate proof of these results of McShane and Tonelli. In addition to being simpler in detail than the previous proofs, the differentiability theorems of $\$ \S 2$ and 3 involve weaker hypotheses than the corresponding theorems of McShane and Tonelli, both in regard to conditions on the integrand function and in regard to the class of arcs considered. Basically, the present proof is intimately related to the proof of the fundamental lemma as given by Bliss [1, pp. 20-21].

2. A general differentiability theorem. Suppose that for $(x, y, r)$ $=\left(x, y_{1}, \cdots, y_{n}, r_{1}, \cdots, r_{n}\right)$ in a region $R$ consisting of all values $(x, y, r)$ satisfying $x_{1} \leqq x \leqq x_{2}, y$ in an open region $\Delta$ of $\left(y_{1}, \cdots, y_{n}\right)$ space, and $r=\left(r_{1}, \cdots, r_{n}\right)$ arbitrary, the integrand function $f(x, y, r)$ satisfies the following conditions:

$\left(\mathrm{H}_{1}\right)$ For fixed values of $(y, r), f(x, y, r)$ is finite and measurable on $x_{1} x_{2}$

$\left(\mathrm{H}_{2}\right)$ For fixed values of $x, f(x, y, r)$ is of class $C^{\prime}$ in $(y, r)$.

We shall be interested in proving a differentiability property of an $\operatorname{arc} y_{i}=y_{i}(x)$ which minimizes $I[y]$ in a class of $\operatorname{arcs} K$. Unquestionably, the case in which $K$ consists of absolutely continuous arcs is the most important. Our argument, however, is not complicated by allowing $K$ to be any prescribed class of arcs which possesses the following properties:

$\left(\mathrm{P}_{0}\right)$ If the arc $y_{i}=y_{i}(x) \quad\left(x_{1} \leqq x \leqq x_{2}\right)$ belongs to $K$, then for each $x$ on $x_{1} x_{2}$ the point $y=\left[y_{i}(x)\right]$ is in the region $\Delta$;

$\left(\mathrm{P}_{1}\right)$ If $y_{i}=y_{i}(x)\left(x_{1} \leqq x \leqq x_{2}\right)$ belongs to $K$, then the functions $y_{i}(x)$ are continuous on $x_{1} x_{2}$ and the derivatives $y_{i}^{\prime}(x)$ exist and are finite a. $e$.

Presented to the Society, April 15, 1939; received by the editors August 29, 1945, and, in revised form, September 28, 1945.

1 Numbers in brackets refer to the Bibliography at the end of the paper. 
(almost everywhere) on this interval;

$\left(\mathrm{P}_{2}\right)$ If $y_{i}=y_{i}(x)\left(x_{1} \leqq x \leqq x_{2}\right)$ belongs to $K$, and the functions $\eta_{i}(x)$ are Lipschitzian on $x_{1} x_{2}$ and satisfy $\eta_{i}\left(x_{1}\right)=0=\eta_{i}\left(x_{2}\right)$, then for a suitably small in absolute value the arc $y_{i}=y_{i}(x)+a \eta_{i}(x)\left(x_{1} \leqq x \leqq x_{2}\right)$ also belongs to $K$.

Actually, in the following proof we use $\left(\mathrm{P}_{2}\right)$ only for sets of functions $\eta_{i}(x)$ for which $n-1$ of these functions are identically zero on $x_{1} x_{2}$.

Along an arc of $K$ the function $f\left(x, y(x), y^{\prime}(x)\right)$ is defined a.e. on $x_{1} x_{2}$. In considering (1.1), and similar integrals, it is to be understood that the integrand is the indicated function where this function is well defined, and equal to zero elsewhere on $x_{1} x_{2}$. Correspondingly, a function $F(x)$ which is defined a.e. on $x_{1} x_{2}$ will be said to be measurable on $x_{1} x_{2}$ if the function which is equal to $F(x)$ at the points where it is defined, and to zero elsewhere on $x_{1} x_{2}$, is measurable on this interval. In view of $\left(\mathrm{H}_{1}\right)$ and $\left(\mathrm{H}_{2}\right)$, and the measurability of a function that is the limit a.e. of a sequence of measurable functions [3, p. 122], it follows that along an arc $y$ of $K$ the function $f$ and its partial derivatives with respect to $y_{j}$ and $r_{j}(j=1, \cdots, n)$ are measurable on $x_{1} x_{2}$.

It is to be noted that we do not suppose that the integral (1.1) exists and is finite for every arc of $K$. An arc $y$ of $K$ is said to minimize (1.1) in this class if $I[y]$ is finite, and for arbitrary arcs $Y$ of $K$ for which $I[Y]$ is finite we have $I[Y] \geqq I[y]$.

The following lemma will be used in the proof of the subsequent theorems.

LEMMA 2.1. Let $\Delta^{*}$ be a given bounded and closed subregion of $\Delta$ in $y$-space, and for a given positive integer $N$ denote by $R_{N}$ the subregion of $R$ consisting of all $(x, y, r)$ satisfying $x_{1} \leqq x \leqq x_{2}, y$ in $\Delta^{*}$, and $\left|r_{i}\right| \leqq N$ $(i=1,2, \cdots, n)$. Then there exists a corresponding function $\psi_{N}(x)$ which is finite and measurable on $x_{1} x_{2}$, and such that for $(x, y, r)$ in $R_{N}$ we have

$$
\begin{aligned}
|f(x, y, r)| \leqq & \psi_{N}(x), \quad\left|f_{y_{i}}(x, y, r)\right| \leqq \\
& \left|f_{r_{i}}(x, y, r)\right| \leqq \psi_{N}(x) \quad(i=1,2, \cdots, n) .
\end{aligned}
$$

In view of hypotheses $\left(\mathrm{H}_{1}\right)$ and $\left(\mathrm{H}_{2}\right)$ it follows that for fixed values of $(y, r)$ the partial derivatives of $f$ with respect to $y_{i}$ and $r_{i}$ $(i=1, \cdots, n)$ are finite and measurable on $x_{1} x_{2}$. Consequently, in $R$ the function

$$
g(x, y, r)=|f(x, y, r)|+\sum_{i=1}^{n}\left[\left|f_{y_{i}}(x, y, r)\right|+\left|f_{r_{i}}(x, y, r)\right|\right]
$$


is continuous in $(y, r)$ for fixed values of $x$ on $x_{1} x_{2}$, and measurable on $x_{1} x_{2}$ for fixed values of $(y, r)$. Now $R_{N}$ is the product of the interval $x_{1} \leqq x \leqq x_{2}$ and the closed and bounded region $D_{N}$ in $(y, r)$ space consisting of all sets $(y, r)$ having $y$ in $\Delta^{*}$ and $\left|r_{i}\right| \leqq N(i=1,2, \cdots, n)$. For each $x$ on $x_{1} x_{2}$ define the functional value $\psi_{N}(x)$ as the maximum of $g(x, y, r)$ on $D_{N}$. In view of the continuity of $g(x, y, r)$ as a function of $(y, r)$ it follows that $\psi_{N}(x)$ is finite on $x_{1} x_{2}$. Moreover, $\psi_{N}(x)$ is measurable on this interval [3, p. 122], since it is the limit superior of a sequence of measurable functions $g\left(x, y^{(k)}, r^{(k)}\right)$, where $\left(y^{(k)}, r^{(k)}\right)$ $(k=1,2, \cdots)$ is a countable sequence dense in $D_{N}$. The function $\psi_{N}(x)$ thus defined clearly satisfies the conclusion of the lemma.

TheOREM 2.1. Suppose that $y_{i}=y_{i}(x)\left(x_{1} \leqq x \leqq x_{2}\right)$ is a minimizing arc for (1.1) in a class $K$, and, in addition to hypotheses $\left(\mathrm{H}_{1}\right)$ and $\left(\mathrm{H}_{2}\right)$, the integrand satisfies with this arc the further condition:

$\left(\mathrm{H}_{3 ; j}\right)$ There exists a constant $\delta>0$ and a function $\phi_{j}(x)$ integrable on $x_{1} x_{2}$ and such that for $\left|\bar{y}_{j}-y_{j}(x)\right|<\delta, \bar{y}_{i}=y_{i}(x)(i \neq j)$, we have

$$
\left|f_{y_{j}}\left(x, \bar{y}, y^{\prime}(x)\right)\right| \leqq \phi_{j}(x)
$$

a.e. on $x_{1} x_{2}$. Then there exists a corresponding constant $c_{j}$ such that

$$
z_{j}(x) \equiv f_{r_{j}}\left(x, y(x), y^{\prime}(x)\right)-\int_{x_{1}}^{x} f_{y_{j}}\left(t, y(t), y^{\prime}(t)\right) d t
$$

is equal to $c_{j}$ a.e. on $x_{1} x_{2}$.

Condition $\left(\mathrm{H}_{3 ; j}\right)$ implies the existence of the integral appearing in the definition of $z_{j}(x)$; it also implies that the integral

$$
\int_{x_{1}}^{x_{2}} f\left(x, Y(x), y^{\prime}(x)\right) d x
$$

exists for all arcs $Y_{i}=Y_{i}(x)$ of $K$ satisfying $\left|Y_{j}(x)-y_{j}(x)\right|<\delta$, $Y_{i}(x)=y_{i}(x)(i \neq j)$ on $x_{1} x_{2}$, since for such arcs,

$$
\left|f\left(x, Y(x), y^{\prime}(x)\right)\right| \leqq\left|f\left(x, y(x), y^{\prime}(x)\right)\right|+\delta \phi_{j}(x) .
$$

Corresponding to a positive integer $N$, let $E_{N}$ denote the set of all points $x$ on $x_{1} x_{2}$ at which the derivatives $y_{i}^{\prime}$ exist and $\left|y_{i}^{\prime}\right| \leqq N-1$ $(i=1, \cdots, n)$. We may suppose that $N$ is sufficiently large to insure that $m\left(E_{N}\right)$, the measure of $E_{N}$, is positive, since $E_{N}$ is a subset of $E_{N+1}(N=1,2, \cdots)$ and $\lim _{N \rightarrow \infty} m\left(E_{N}\right)=x_{2}-x_{1}$. Furthermore, let the region $\Delta^{*}$ of Lemma 2.1 be such that it contains in its interior all the points $y=\left[y_{i}(x)\right]\left(x_{1} \leqq x \leqq x_{2}\right)$ belonging to the supposed minimizing arc, and denote by $E_{N ; M}$ the subset of $E_{N}$ where the function $\psi_{N}(x)$ 
of that lemma satisfies the condition $\psi_{N}(x) \leqq M(M=1,2, \cdots)$. Since $E_{N ; M}$ is a subset of $E_{N ; M+1}$ and $\lim _{M \rightarrow \infty} m\left(E_{N ; M}\right)=m\left(E_{N}\right)>0$, we may also assume that $M$ is so chosen that $m\left(E_{N ; M}\right)>0$.

Now on $E_{N ; M}$ the function $z_{j}(x)$ defined in $\left(\mathrm{H}_{3 ; j}\right)$ is bounded and measurable, hence integrable. Consequently, there is a unique constant $c_{j}$ such that

$$
0=\int_{E_{N ; \boldsymbol{M}}}\left[z_{j}(x)-c_{j}\right] d x=\int_{B_{N_{i} \boldsymbol{M}}} z_{j}(x) d x-c_{j} m\left(E_{N ; M}\right) .
$$

Now define $\eta_{i}(x) \equiv 0$ for $i \neq j$, and

$$
\eta_{j}(x)=\int_{x_{1}}^{x}\left[z_{j}(t)-c_{j}\right] I_{N ; M} d t,
$$

where $I_{N ; M}$ is the characteristic function of the set $E_{N ; M}$. The functions $\eta_{i}(x)$ are clearly Lipschitzian on $x_{1} x_{2}$ and satisfy $\eta_{i}\left(x_{1}\right)=0$ $=\eta_{i}\left(x_{2}\right)$; moreover, $\eta_{i}^{\prime}=0$ a.e. on $C E_{N ; M}$, the complement of $E_{N ; M}$ on $x_{1} x_{2}$. In view of the Lipschitzian character of the functions $\eta_{i}(x)$ and property $\left(\mathrm{P}_{2}\right)$, there exists a positive constant $a_{0}$ such that for $|a|<a_{0}$ the arc $y_{i}=y_{i}(x)+a \eta_{i}(x)$ belongs to $K$ and lies in the region $\Delta^{*}$ of Lemma 2.1. We shall also suppose that $a_{0}$ is chosen so small that $a_{0}\left|\eta_{j}(x)\right|<\delta$ on $x_{1} x_{2}$ and $a_{0}\left|\eta_{j}^{\prime}(x)\right| \leqq 1$ a.e. on this interval. In particular, we have that $\left|y_{j}^{\prime}(x)+a \eta_{j}^{\prime}(x)\right| \leqq N$ a.e. on $E_{N}$, and hence a.e. on the subset $E_{N ; M}$. For such values of $a$ the integral $I[y+a \eta]$ exists and is finite, and

$$
\begin{aligned}
I[y+a \eta]= & \int_{C E_{\mathbb{N}_{i} \boldsymbol{X}}} f\left(x, y+a \eta, y^{\prime}\right) d x \\
& +\int_{\mathbb{E}_{N_{i}: \mathcal{X}}} f\left(x, y+a \eta, y^{\prime}+a \eta^{\prime}\right) d x .
\end{aligned}
$$

The existence and finiteness of the first integral of (2.6) follows from (2.3) for $Y(x)=y(x)+a \eta(x)$. The existence and finiteness of the second integral of (2.6) follows from the measurability of the integrand, together with the fact that a.e. on $E_{N ; M}$ the set $\left(x, y(x)+a \eta(x), y^{\prime}(x)\right.$ $\left.+a \eta^{\prime}(x)\right)$ is in the region $R_{N}$ of Lemma 2.1 , and consequen tly $\left|f\left(x, y(x)+a \eta(x), y^{\prime}(x)+a \eta^{\prime}(x)\right)\right| \leqq \psi_{N}(x) \leqq M$ on this set.

Now, in view of $\left(\mathrm{H}_{3 ; j}\right)$, for $|a|<a_{0}$ the partial derivative of $f\left(x, y(x)+a \eta(x), y^{\prime}(x)\right)$ with respect to $a$ exists and does not exceed in absolute value the integrable function $\left|\eta_{j}(x)\right| \phi_{j}(x)$, which, in turn, is not greater than $\left(\delta / a_{0}\right) \phi_{j}(x)$. Moreover, a.e. on $E_{N ; M}$ the partial derivative of $f\left(x, y(x)+a \eta(x), y^{\prime}(x)+a \eta^{\prime}(x)\right)$ with respect to $a$ exists 
and does not exceed in absolute value $\left(\left|\eta_{j}(x)\right|+\left|\eta_{i}^{\prime}(x)\right|\right) \psi_{N}(x)$ $\leqq\left[(\delta+1) / a_{0}\right] M$. It then follows $[3$, p. 216] that the derivative of (2.6) with respect to $a$ exists for $|a|<a_{0}$, and is given by the usual differentiation under the integral sign. For $a=0$ this derivative is

$$
\begin{aligned}
\int_{C E_{N_{i}, \mu}} f_{y_{j} \eta_{j}} d x & +\int_{E_{N_{i}: \mu}}\left[f_{y_{j} \eta_{j}}+f_{r_{j} \eta_{j}^{\prime}}\right] d x \\
& =\int_{x_{1}}^{x_{2}}\left[f_{y_{j} \eta_{j}}+f_{r_{j} \eta_{j}^{\prime}}\right] d x=\int_{x_{1}}^{x_{2}} z_{j} \eta_{j}^{\prime} d x,
\end{aligned}
$$

where the arguments of the partial derivatives of $f$ occurring in (2.7) are $\left(x, y(x), y^{\prime}(x)\right)$. The last relation follows by the usual integration by parts, and use of the conditions $\eta_{j}\left(x_{1}\right)=0=\eta_{j}\left(x_{2}\right)$. Since the minimizing property of $y$ requires that this derivative be zero, and as the integral of $\eta_{1}^{\prime}(x)$ over $x_{1} x_{2}$ is equal to zero, it follows, in particular, that

$$
\begin{aligned}
0=\int_{x}^{x_{2}}\left[z_{j}(x)-c_{j}\right] \eta_{j}^{\prime}(x) d x & =\int_{x_{1}}^{x_{2}}\left[z_{j}(x)-c_{j}\right]^{2} I_{N ; M} d x \\
& =\int_{E_{N_{i}, K}}\left[z_{j}(x)-c_{j}\right]^{2} d x .
\end{aligned}
$$

From (2.8) we have that $z_{j}(x)=c_{j}$ a.e. on $E_{N ; M}$. Now the constant $c_{j}$ is seemingly dependent on $N$ and $M$. Since, however, $E_{N ; M}$ is a subset of $E_{N ; M+1}(M=1,2, \cdots)$ and $\lim _{M \rightarrow \infty} m\left(E_{N ; M}\right)=m\left(E_{N}\right)$, it is seen that a.e. on $E_{N}$ we have $z_{j}(x)=c_{j}$, where $c_{j}$ is the constant determined by any set $E_{N ; M}$ for which $m\left(E_{N ; M}\right)>0$. Similarly, since $E_{N}$ is a subset of $E_{N+1}(N=1,2, \cdots)$ and $\lim _{N \rightarrow \infty} m\left(E_{N}\right)=x_{2}-x_{1}$, it follows that $z_{j}(x)=c_{j}$ a.e. on $x_{1} x_{2}$.

3. A second differentiability theorem. We shall now prove another differentiability property of a minimizing arc for (1.1) under the further hypotheses:

(H) $f(x, y, r)$ is of class $C^{\prime}$ in all its arguments on $R$;

$\left(\mathrm{P}_{2}^{\prime}\right)$ If $y_{i}=y_{i}(x)\left(x_{1} \leqq x \leqq x_{2}\right)$ is an arc of $K$, and the function $\zeta(x)$ is such that $\zeta\left(x_{1}\right)=x_{1}, \zeta\left(x_{2}\right)=x_{2}$, while there is a constant $k \geqq 1$ such that $\left(x^{\prime}-x^{\prime \prime}\right) / k \leqq \zeta\left(x^{\prime}\right)-\zeta\left(x^{\prime \prime}\right) \leqq k\left(x^{\prime}-x^{\prime \prime}\right)$ for arbitrary values $x^{\prime}, x^{\prime \prime}$ satisfying $x_{1} \leqq x^{\prime \prime}<x^{\prime} \leqq x_{2}$, then $y_{i}=y_{i}(\zeta(x))$ is also an arc of $K$.

Condition $\left(\mathrm{P}_{2}^{\prime}\right)$ is satisfied if $K$ is either the class of arcs of bounded variation, the class of absolutely continuous arcs, or the class of Lipchitzian arcs.

THEOREM 3.1. Suppose that $y_{i}=y_{i}(x)\left(x_{1} \leqq x \leqq x_{2}\right)$ is a minimizing 
arc for $(1.1)$ in a class $K$ satisfying $\left(\mathrm{P}_{0}\right),\left(\mathrm{P}_{1}\right)$ and $\left(\mathrm{P}_{2}^{\prime}\right)$, and, in addition to hypothesis $(\mathrm{H})$, the integrand satisfies with this arc the further condition:

$\left(\mathrm{H}_{3 ; 0}\right)$ There exists a constant $\delta$ and a function $\phi_{0}(x)$ integrable on $x_{1} x_{2}$ and such that if $x$ and $\bar{x}$ are on this interval and satisfy $|\bar{x}-x|<\delta$, then a.e. on $x_{1} x_{2}$,

$$
\left|f_{x}\left(\bar{x}, y(x), y^{\prime}(x)\right)\right| \leqq \phi_{0}(x)
$$

Then there exists a constant $c_{0}$ such that

$$
\begin{gathered}
z(x) \equiv f\left(x, y(x), y^{\prime}(x)\right)-\sum_{i=1}^{n} y_{i}^{\prime}(x) f_{r_{i}}\left(x, y(x), y^{\prime}(x)\right) \\
-\int_{x_{1}}^{x} f_{x}\left(t, y(t), y^{\prime}(t)\right) d t
\end{gathered}
$$

is equal to $c_{0}$ a.e. on $x_{1} x_{2}$.

Condition $\left(\mathrm{H}_{3 ; 0}\right)$ implies, in particular, that the partial derivative $f_{x}\left(x, y(x), y^{\prime}(x)\right)$ is integrable on $x_{1} x_{2}$. Corresponding to a positive integer $N$, again let $E_{N}$ denote the subset of $x_{1} x_{2}$ on which the derivatives $y_{i}^{\prime}(x)$ exist and satisfy $\left|y_{i}^{\prime}(x)\right| \leqq N-1(\imath=1, \cdots, n)$. Hypothesis $(\mathrm{H})$ implies that along the minimizing arc the integrand $f$ and its partial derivatives with respect to $r_{i}(i=1, \cdots, n)$ are bounded functions of $x$ on $E_{N}$. As in the proof of Theorem 2.1, we may suppose that $N$ is so large that $m\left(E_{N}\right)>0$. Now on $E_{N}$ the function $z(x)$ is bounded and measurable, and hence integrable. Let $c_{0}$ be the constant determined by the condition

$$
0=\int_{E_{N}}\left[z(x)-c_{0}\right] d x=\int_{E_{N}} z(x) d x-c_{0} m\left(E_{N}\right),
$$

and set

$$
\eta(x)=\int_{x_{1}}^{x}\left[z(t)-c_{0}\right] I_{N} d t,
$$

where $I_{N}$ is the characteristic function of the set $E_{N}$. Then $\eta(x)$ is Lipschitzian on $x_{1} x_{2}, \eta\left(x_{1}\right)=0=\eta\left(x_{2}\right)$, and for $a_{0}$ sufficiently small the function $T(t ; a)=t+a \eta(t)$ is such that $\left(t^{\prime}-t^{\prime \prime}\right) / 2 \leqq T\left(t^{\prime} ; a\right)-T\left(t^{\prime \prime} ; a\right)$ $\leqq 2\left(t^{\prime}-t^{\prime \prime}\right)$ for all values of $a$ such that $|a|<a_{0}$, and for arbitrary $t^{\prime}, t^{\prime \prime}$ satisfying $x_{1} \leqq t^{\prime \prime}<t^{\prime} \leqq x_{2} ;$ moreover, $T\left(x_{1} ; a\right)=x_{1}, T\left(x_{2} ; a\right)=x_{2}$. Hence for such values of $a$ the equation

$$
x=t+a \eta(t) \equiv T(t ; a)
$$

has a unique solution $t=\zeta(x ; a)$, which is Lipschitzian on $x_{1} x_{2}$ and 
such that $\left(x^{\prime}-x^{\prime \prime}\right) / 2 \leqq \zeta\left(x^{\prime} ; a\right)-\zeta\left(x^{\prime \prime} ; a\right) \leqq 2\left(x^{\prime}-x^{\prime \prime}\right)$ for arbitrary values $x^{\prime}, x^{\prime \prime}$ satisfying $x_{1} \leqq x^{\prime \prime}<x^{\prime} \leqq x_{2}$. We have also that $\zeta\left(x_{1}, a\right)$ $=x_{1}, \zeta\left(x_{2}, a\right)=x_{2}$, while $\zeta(x ; 0) \equiv x$ on $x_{1} x_{2}$. It then follows from $\left(\mathrm{P}_{2}^{\prime}\right)$ that $y_{i}(x ; a)=y_{i}(\zeta(x ; a))\left(x_{1} \leqq x \leqq x_{2}\right)$ defines an arc of $K$ such that $y_{i}(x ; 0)=y_{i}(x)$.

From known theorems on the change of variable in a simple in. tegral $[3$, p. 215] we then have that the integral (1.1) calculated along the arc $y_{i}=y_{i}(x ; a)$ is finite and equal to

$$
\begin{aligned}
\int_{x_{1}}^{x_{2}} f(t & \left.+a \eta(t), y(t), y^{\prime}(t) /\left[1+a \eta^{\prime}(t)\right]\right)\left[1+a \eta^{\prime}(t)\right] d t \\
= & \int_{E_{N}} f\left(t+a \eta(t), y(t), y^{\prime}(t) /\left[1+a \eta^{\prime}(t)\right]\right)\left[1+a \eta^{\prime}(t)\right] d t \\
& +\int_{C E_{N}} f\left(t+a \eta(t), y(t), y^{\prime}(t)\right) d t
\end{aligned}
$$

Since $\left|y_{i}^{\prime}\right| \leqq N-1$ on $E_{N}$, it follows from $(\mathrm{H})$ that a.e. on $E_{N}$ the partial derivative with respect to $a$ of the integrand of the first integral of (3.1) exists for $|a|<a_{0}$; moreover, this partial derivative is uniformly bounded for $x$ on this subset of $E_{N}$ and $|a|<a_{0}$. Hypotheses $(\mathrm{H})$ and $\left(\mathrm{H}_{8 ; 0}\right)$ imply that for sufficiently small values of $a$ the partial derivative with respect to $a$ of the integrand of the second integral exists and does not exceed in absolute value the integrable function $|\eta(t)| \phi_{0}(t)$. It then follows from the previously applied theorem on differentiability of integrals that the derivative of (3.1) exists for $a=0$, and is given by

$$
\int_{x_{1}}^{x_{2}}\left[\left(f-\sum_{i=1}^{n} y_{i}^{\prime} f_{r_{0}}\right) \eta^{\prime}+f_{x} \eta\right] d x
$$

where the arguments of $f$ and its partial derivative occurring in (3.2) are $\left(x, y(x), y^{\prime}(x)\right)$.

In view of the minimizing property of the arc $y_{i}=y_{i}(x)$, the integrability of $f_{x}$ along this arc, and the conditions $\eta\left(x_{1}\right)=0=\eta\left(x_{2}\right)$, we conclude that

$$
\begin{aligned}
0 & =\int_{x_{1}}^{m_{2}} z(x) \eta^{\prime}(x) d x=\int_{x_{1}}^{x_{1}}\left[z(x)-c_{0}\right] \eta^{\prime}(x) d x \\
& =\int_{x_{1}}^{x_{2}}\left[z(x)-c_{\theta}\right]^{2} I_{N} d x=\int_{B_{N}}\left[z(x)-c_{0}\right]^{2} d x .
\end{aligned}
$$

That is, $z(x)=c_{0}$ a.e. on $E_{N}$. As $E_{N}$ is a subset of $E_{N+1}(N=1,2, \cdots)$ 
and $\lim _{N \rightarrow \infty} m\left(E_{N}\right)=x_{2}-x_{1}$, it then follows that $z(x)=c_{0}$ a.e. on $x_{1} x_{2}$.

4. Further remarks. The general differentiability theorems of $\$ \S 2$ and 3 involve, respectively, hypotheses $\left(\mathrm{H}_{3 ; i}\right)$ and $\left(\mathrm{H}_{3 ; 0}\right)$ for the considered minimizing arc. Now suppose that the integrand function $f(x, y, r)$ satisfies $\left(\mathrm{H}_{1}\right)$ and $\left(\mathrm{H}_{2}\right)$, and, in addition to $\left(\mathrm{P}_{0}\right),\left(\mathrm{P}_{1}\right)$ and $\left(\mathrm{P}_{2}\right)$, the class $K$ contains only arcs $y_{i}=y_{i}(x)$ such that the derivative functions $y_{i}^{\prime}(x)$ are integrable on $x_{1} x_{2}$. Then every arc of $K$ for which $I[y]$ is finite satisfies $\left(\mathrm{H}_{3 ; i}\right)$ whenever the integrand function satisfies the following condition:

$\left(\mathrm{H}_{3, j}^{*}\right)$ There exist positive constants $M_{1}, M_{2}, \delta$ such that if $(x, y, r)$ and $(x, \bar{y}, r)$ are points of $R$ with $\left|\bar{y}_{j}-y_{j}\right|<\delta, \bar{y}_{i}=y_{i}(i \neq j)$ then

$$
\left|f_{y_{j}}(x, \bar{y}, r)\right| \leqq M_{1}|f(x, y, r)|+M_{2}\left[1+\sum_{i} r_{i}^{2}\right]^{1 / 2} .
$$

Correspondingly, if $f(x, y, r)$ satisfies $(\mathrm{H})$, and, in addition to $\left(\mathrm{P}_{0}\right)$, $\left(\mathrm{P}_{1}\right)$ and $\left(\mathrm{P}_{2}^{\prime}\right)$, the class $K$ contains only arcs $y_{i}=y_{i}(x)$ such that the functions $y_{i}^{\prime}(x)$ are integrable on $x_{1} x_{2}$, then every arc of $K$ for which $I[y]$ is finite satisfies $\left(\mathrm{H}_{3 ; 0}\right)$ under the following additional hypothesis:

$\left(\mathrm{H}_{3 ; 0}^{*}\right)$ There exist positive constants $M_{1}, M_{2}, \delta$ such that if $(x, y, r)$ and $(\bar{x}, y, r)$ are points of $R$ with $|\bar{x}-x|<\delta$, then

$$
\left|f_{x}(\bar{x}, y, r)\right| \leqq M_{1}|f(x, y, r)|+M_{2}\left[1+\sum_{i} r_{i}^{2}\right]^{1 / 2} .
$$

The above hypotheses $\left(\mathrm{H}_{3 ; j}^{*}\right)$ and $\left(\mathrm{H}_{3 ; 0}^{*}\right)$ have been used by McShane [2]. In connection with the above remarks it is to be noted that if the term $M_{2}\left[1+\sum_{i} r_{i}^{2}\right]^{1 / 2}$ in (4.1) and (4.2) is replaced by $M_{2}$, then the condition that the functions $y_{i}^{\prime}(x)$ be integrable on $x_{1} x_{2}$ is not needed to insure that every arc of $K$ for which $I[y]$ is finite will satisfy the corresponding condition $\left(H_{3 ; j}\right)$ or $\left(\mathrm{H}_{3 ; 0}\right)$.

Finally, it is to be remarked that as a very special case of Theorem 2.1 one has the following generalization of the fundamental lemma of the calculus of variations:

If $M(x)$ is finite and measurable on $x_{1} x_{2}$, and the integral

$$
\int_{x_{1}}^{x_{2}} M(x) \eta^{\prime}(x) d x
$$

is zero for every Lipschitzian function $\eta(x)$ satisfying $\eta\left(x_{1}\right)=0=\eta\left(x_{2}\right)$, and for which the above integral exists and is finite, then there exists a constant $c$ such that $M(x)=c$ a.e. on $x_{1} x_{2}$. 


\section{BIBLIOGRAPHY}

1. Bliss, G. A., Calculus of variations, Carus Mathematical Monograph, no. 1, Open Court Publishing Co., Chicago, 1925.

2. McShane, E. J., The du Bois-Reymond relation in the calculus of variations, Math. Ann. vol. 109 (1934) pp. 746-755.

3. - Integration, Princeton University Press, 1944.

4. Tonelli, L., Sulle proprieta delle estremanti, Annali della R. Scuola Normale Superiore di Pisa (2) vol. 3 (1934) pp. 213-237.

NORTHWESTERN UNIVERSITY 